\title{
Monotone maps of $G$-like continua with positive topological entropy yield indecomposability
}

\author{
Hisao Kato \\ Institute of Mathematics, University of Tsukuba, Ibaraki 305-8571, Japan
}

\begin{abstract}
In the previous paper [5], we proved that if for any graph $G$, a homeomorphism on a $G$-like continuum $X$ has positive topological entropy, then the continuum $X$ contains an indecomposable subcontinuum. Also, if for a tree $G$, a monotone map on a $G$-like continuum $X$ has positive topological entropy, then the continuum $X$ contains an indecomposable subcontinuum. In this note, we extend these results. In fact, we prove that if for any graph $G$, a monotone map on a $G$-like continuum $X$ has positive topological entropy, then the continuum $X$ contains an indecomposable subcontinuum. Also we study topological entropy of monotone maps on Suslinean continua.
\end{abstract}

\section{Introduction}

During the last thirty years or so, many interesting connections between topological dynamics and continuum theory have been studied by many authors. In particular, we are interested in the fact that several complicated dynamics should imply existence of complicated continua and in many cases, such continua are indecomposable continua which are central subjects in continuum theory (see the references $[1,2,5-19,23-26,28,29]$ ).

A continuum is a compact connected metric space. We say that a continuum is nondegenerate if it has more than one point. A continuum is indecomposable $([21,22,27])$ if it is nondegenerate and it is not the union of two proper subcontinua. A continuum $H$ is an $n$-od $(2 \leq n<\infty)$ if $H$ contains a subcontinuum $A$ such that the complement of $A$ in $H$ is the union $n$ nonempty mutually separated sets. For any continuum $X$, let

$$
T(X)=\sup \{n \mid \text { there is an } n \text {-od in } X\} .
$$

For any continuum $Z$, the set $Z(p)$ of all points of the continuum $Z$, which can be joined with the point $p$ by a proper subcontinuum of $Z$, is said to be the composant of the point $p \in Z$ (see [21, p.208]). It is well known that if $Z$ is an indecomposable continuum, the family $\{Z(p) \mid p \in Z\}$ of all composants of $Z$ is a family of uncountable mutually disjoint sets $Z(p)$ which are dense $F_{\sigma}$-sets in $Z$ (see [21, p.212, Theorem 6]).

In this note, all maps are assumed to be continuous. Let $\mathbb{N}$ be the set of natural numbers. A map $g$ from $X$ onto $G$ is an $\epsilon$-map $(\epsilon>0)$ if for every $y \in G$, the diameter of $g^{-1}(y)$ is less than $\epsilon$. A continuum $X$ is $G$-like if for every $\epsilon>0$ there is an $\epsilon$-map from $X$ onto $G$. It is easy to see that if a continuum $X$ is $G$-like for some graph $G$ (=1-dimensional connected compact polyhedron), then $T(X)<\infty$. Our focus in this article is on $G$-like continua where $G$ is a graph. A surjectve map $f: X \rightarrow Y$ is a monotone map if $f^{-1}(y)$ is connected for each $y \in Y$. We recall that this definition is equivalent to stating that the preimage of a continuum is a continuum whenever nonempty.

\footnotetext{
${ }^{1} 2010$ Mathematics Subject Classification: Primary 37B45, 37B40; Secondary 54H20, 54F15

${ }^{2}$ Key words and phrases: Topological entropy, continuum, indecomposable, monotone map, inverse limit, $G$-like continuum
} 
If $f: X \rightarrow X$ is a map, then we use $\lim (X, f)$ to denote the inverse limit of $X$ with $f$ as the bonding maps, i.e.,

$$
\lim _{\longleftarrow}(X, f)=\left\{\left(x_{i}\right) \in X^{\mathbb{N}} \mid f\left(x_{i+1}\right)=x_{i}\right\} \subset X^{\mathbb{N}}
$$

The topology on $\lim (X, f)$ is the subspace topology inherited from the product topology on $X^{\mathbb{N}}$. In particular, if $X$ is compact, then $\lim (X, f)$ is compact and, similarly, if $X$ is a continuum, then so is $\lim _{(}(X, f)$. The reader may refer to [21] and [27] for standard facts concerning continuum theory.

\section{Monotone maps of continua and topological entropy}

Let $X$ be a compact metric space and $\mathcal{U}, \mathcal{V}$ be two covers of $X$. Put

$$
\mathcal{U} \vee \mathcal{V}=\{U \cap V: U \in \mathcal{U}, V \in \mathcal{V}\}
$$

The quantity $N(\mathcal{U})$ denotes minimal cardinality of subcover of $\mathcal{U}$. Let $f: X \rightarrow X$ be a map and let $\mathcal{U}$ be an open cover of $X$. Put

$$
h(f, \mathcal{U})=\lim _{n \rightarrow \infty} \frac{\log N\left(\mathcal{U} \vee f^{-1}(\mathcal{U}) \vee \ldots \vee f^{-n+1}(\mathcal{U})\right)}{n} .
$$

The topological entropy of $f$, denoted by $h(f)$, is the supremum of $h(f, \mathcal{U})$ for all open covers $\mathcal{U}$ of $X$. The reader may refer to $[3,4,20,23]$ for important facts concerning topological entropy.

In [5], we obtained the following theorem.

Theorem 2.1. ([5]) Let $G$ be any graph. If a homeomorphism $f$ on a $G$-like continuum $X$ has positive topological entropy, i.e., $h(f)>0$, then $X$ contains an indecomposable subcontinuum.

We will prove the following main theorem which is an extension of Theorem 2.1.

Theorem 2.2. Let $G$ be any graph. If a monotone map $f$ on a $G$-like continuum $X$ has positive topological entropy, then $X$ contains an indecomposable subcontinuum.

To prove Theorem 2.2, we need the following lemma which is a result of continuum theory.

Lemma 2.3. Let $X$ and $Y$ be continua with $T(X)<\infty$. If $f: X \rightarrow Y$ is an (onto) monotone map and $Z$ is an indecomposable subcontinuum of $X$ such that $f(Z)$ is nondegenerate, then $f(Z)$ is an indecomposable subcontinuum of $Y$.

Proof. Since $f: X \rightarrow Y$ is monotone, we see that $T(Y) \leq T(X)<\infty$. For each $x \in Z$, let $Z(x)$ denote the composant of $Z$ containing $x \in Z$. Let $\operatorname{Comp}(Z)$ be the set of all composants of $Z$. We take a subset $\left\{x_{\alpha} \in Z \mid \alpha \in \Lambda\right\}$ of $Z$ such that $Z\left(x_{\alpha}\right) \cap Z\left(x_{\beta}\right)=\emptyset$ for $\alpha \neq \beta$ and

$$
\operatorname{Comp}(Z)=\left\{Z\left(x_{\alpha}\right) \mid \alpha \in \Lambda\right\},
$$

where $\Lambda$ is an index set which is uncountable. 
For each $\alpha, \beta \in \Lambda$, we define $\alpha \sim_{f} \beta$ provided that $f\left(Z\left(x_{\alpha}\right)\right) \cap f\left(Z\left(x_{\beta}\right)\right) \neq \emptyset$. Also, for each $\alpha, \beta \in \Lambda$, we define $\alpha \sim \beta$ provided that there is a finite sequence $\alpha=\alpha_{1}, \alpha_{2}, \ldots, \alpha_{s}=\beta$ of $\Lambda$ such that $\alpha_{i} \sim_{f} \alpha_{i+1}$ for each $i=1,2, . ., s-1$. Then the relation $\sim$ is an equivalence relation on $\Lambda$. Note that $f\left(Z\left(x_{\alpha}\right)\right) \cap f\left(Z\left(x_{\beta}\right)\right) \neq \emptyset$ if and only if there is a point $y \in Y$ with $f^{-1}(y) \cap Z\left(x_{\alpha}\right) \neq \emptyset \neq f^{-1}(y) \cap Z\left(x_{\beta}\right)$. We will show that for each $\alpha \in \Lambda$, the cardinality $|[\alpha]|$ of the set $[\alpha]=\{\beta \in \Lambda \mid \alpha \sim \beta\}$ is $\leq T(X)$. Suppose, on the contrary, that for some $\alpha \in \Lambda$, $|[\alpha]| \geq n=T(X)+1$. Let $\Lambda(n)$ be any subset of $[\alpha]$ with $|\Lambda(n)|=n$. Note that for each $\beta \in \Lambda(n)$, we can find a sequence $x_{\alpha}=a_{1}, \tilde{a}_{1}, a_{2}, \tilde{a}_{2}, \ldots, a_{s(\beta)}, \tilde{a}_{s(\beta)}=x_{\beta}$ of points of $Z$ such that for each $i$,

(1) $a_{i}$ and $\tilde{a}_{i}$ are contained in the same composant of $Z$, i.e., $Z\left(a_{i}\right)=Z\left(\tilde{a}_{i}\right)$,

(2) $Z\left(\tilde{a}_{i}\right) \neq Z\left(a_{i+1}\right)$ and

(3) $f\left(\tilde{a}_{i}\right)=f\left(a_{i+1}\right)$.

For each $i$, we can choose a subcontinuum $C\left(a_{i}, \tilde{a}_{i}\right)$ in the composant $Z\left(a_{i}\right)$ containing $a_{i}$ and $\tilde{a}_{i}$ and we put

$$
A_{\beta}=\bigcup_{i=1}^{s(\beta)} C\left(a_{i}, \tilde{a}_{i}\right) \cup \bigcup_{i=1}^{s(\beta)-1} f^{-1}\left(f\left(\tilde{a}_{i}\right)\right) .
$$

Also, we put

$$
A=\bigcup_{\beta \in \Lambda(n)} A_{\beta}
$$

Since $C\left(a_{i}, \tilde{a}_{i}\right)$ is a continuum containing $a_{i}, \tilde{a}_{i}$ and $f^{-1}\left(f\left(\tilde{a}_{i}\right)\right)$ is a continuum containing $\tilde{a}_{i}, a_{i+1}$, we see that $A_{\beta}$ is a continnum containing $x_{\alpha}$ and $x_{\beta}$. Since $x_{\alpha} \in \bigcap_{\beta \in \Lambda(n)} A_{\beta}$, we see that $A$ is also a continuum. Since $Z$ is indecomposable, we see that each $C\left(a_{i}, \tilde{a}_{i}\right)$ has the empty interior in $Z$ and hence $Z-A$ is a nonempty open set of $Z$. Since each $Z\left(x_{\beta}\right)(\beta \in \Lambda(n))$ is dense in $Z$, we can find a continuum $B_{\beta}$ in $Z\left(x_{\beta}\right)$ such that $B_{\beta}-A \neq \emptyset$ and $B_{\beta} \cap A \neq \emptyset$. Note that the family $\left\{B_{\beta} \mid \beta \in \Lambda(n)\right\}$ is a family of mutually disjoint sets. Let

$$
H=\bigcup_{\beta \in \Lambda(n)} B_{\beta} \cup A
$$

Note that $H$ is a continuum which contains a subcontinuum $A$ such that the complement of $A$ in $H$ is the union of $n$ nonempty mutually separated sets $B_{\beta}-A(\beta \in \Lambda(n))$. Hence $H$ is an $n$-od in X. This is a contradiction. Hence $|[\alpha]| \leq T(X)<\infty$.

Note that $\Lambda$ is an uncountable set. By the fact that $|[\alpha]|$ is finite for each $\alpha \in \Lambda$, we can choose an uncountable subset $\Lambda^{\prime}$ of $\Lambda$ such that the family $\left\{f\left(Z\left(x_{\alpha}\right)\right) \mid \alpha \in \Lambda^{\prime}\right\}$ is a family of mutually disjoint subsets of $f(Z)$.

Finally, we will prove that $f(Z)$ is indecomposable. Suppose, on the contrary, that $f(Z)$ is decomposable. There is a proper subcontinuum $A$ of $f(Z)$ with $\operatorname{Int}_{f(Z)}(A) \neq \emptyset$. Since each composant of $Z$ is dense in $Z$, for any $\alpha_{i} \in \Lambda^{\prime}(i=1,2, \ldots, T(Y)+1)$ there is a subcontinuum $B_{i}$ of $f\left(Z\left(x_{\alpha_{i}}\right)\right)$ such that $B_{i} \cap A \neq \emptyset$ and $B_{i}-A \neq \emptyset$ for each $i$. Then $Y$ contains a $(T(Y)+1)$-od

$$
A \cup \bigcup_{i=1}^{T(Y)+1} B_{i} .
$$

This is a contradiction. Consequently, we see that $f(Z)$ is an indecomposable subcontinuum of $Y$. 
Now, we will prove Theorem 2.2 .

Proof. Since $X$ is $G$-like, then $\lim (X, f)$ is also $G$-like, and hence we see that $T(X)<\infty$ and $T(\lim (X, f))<\infty$. Consider the shift homeomorphism $\sigma_{f}: \lim (X, f) \rightarrow \lim (X, f)$ defined by $\sigma_{f}\left(\left(x_{1}, x_{2}, \ldots\right)\right)=\left(f\left(x_{1}\right), f\left(x_{2}\right), \ldots\right)\left(=\left(f\left(x_{1}\right), x_{1}, x_{2}, \ldots\right)\right)$. Note that the topological entropy of $f$ is equal to the topological entropy of $\sigma_{f}$. By [5, Theorem 5.5], there is an indecomposable

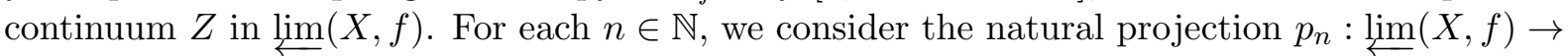
$X$ defined by $p_{n}\left(\left(x_{1}, x_{2}, \ldots, x_{n}, x_{n+1}, \ldots\right)\right)=x_{n}$. Note that for $i \in \mathbb{N}, f^{i}$ is also monotone (e.g. see $[27,(8.46)])$ and for $y \in X$,

$$
p_{n}^{-1}(y)=\lim _{\longleftarrow}(X, f) \cap\left(\left\{f^{n-1}(y)\right\} \times\left\{f^{n-2}(y)\right\} \times \cdots \times\{f(y)\} \times\{y\} \times f^{-1}(y) \times f^{-2}(y) \times \cdots\right) .
$$

Therefore $p_{n}^{-1}(y)$ can be represented by an inverse limit of continua. Hence $p_{n}^{-1}(y)$ is a continuum and so $p_{n}$ is monotone. We can choose a sufficiently large natural number $n \in \mathbb{N}$ such that $p_{n}(Z)$ is nondegenerate. By the above lemma, we see that $p_{n}(Z)$ is an indecomposable subcontinuum of $X$.

For uniform positive topological entropy (see [5, Corollary 5.6]), we have the following.

Corollary 2.4. Let $G$ be any graph. If a monotone map $f$ on a $G$-like continuum $X$ has uniform positive topological entropy, then the continuum $X$ is itself an indecomposable continuum.

A continuum $X$ is hereditarily decomposable if $X$ does not contain any indecomposable subcontinua. A continuum $X$ is Suslinean if $X$ has no uncountable mutually disjoint nondegenerate subcontinua. Note that Suslinean continua are herediterily decomposable. A continuum $X$ is regular if $X$ has an open base $\left\{U_{i} \mid i \in \mathbb{N}\right\}$ such that the boundary $B d\left(U_{i}\right)$ of $U_{i}$ is a finite set. A continuum $X$ is rational if $X$ has an open base $\left\{U_{i} \mid i \in \mathbb{N}\right\}$ such that the boundary $B d\left(U_{i}\right)$ of $U_{i}$ is a countable set. Note that all rational continua are Suslinean continua.

Remark. (1) In Lemma 2.3, for a 1-dimensional continuum $X$ we can not omit the condition $T(X)<\infty$. In fact, it is known that for any Peano continuum $Y$ there exists a monotone map from the Menger curve $M$ onto $Y$. Hence there is a monotone map $f: M(=X) \rightarrow[0,1]$ and we can take an indecomposable subcontinuum $Z$ of $M$ such that $f(Z)$ is a nondegenerate interval. (2) There is a regular curve $X$ with $T(X)=\infty$. For example, Wazewski's universal dendrite $X$ is a regular curve with $T(X)=\infty$ (see $[27,(10.37)])$.

(3) There is a 1-dimensional continuum $X$ with $T(X)=\infty$ such that $X$ is hereditarily decomposable, non-Suslinean and $X$ admits a homeomorphism with positive topological entropy. For example, the topological cone $X=\operatorname{Cone}(C)$ of a Cantor set $C$ is such a continuum (see [27, $(3.15)])$.

In $[28,8]$, we obtained the following result.

Theorem 2.5. ([28, 8]) Any monotone map on a regular continuum has zero topological entropy.

In [26], Mouron proved the following.

Theorem 2.6. ([26]) Let $X$ be a Suslinean continuum. Then any homeomorphism on $X$ has zero topological entropy. In particular, any homeomorphism on a rational continuum has zero topological entropy. 
Theorem 2.2 implies the following.

Corollary 2.7. Let $G$ be any graph and let $X$ be a $G$-like continuum which is hereditarily decomposable. Then any monotone map on $X$ has zero topological entropy.

We will prove the following.

Theorem 2.8. Let $X$ be a Suslinean continuum with $T(X)<\infty$. Then any monotone map on $X$ has zero topological entropy.

Proof. The proof is similar to the proofs of Lemma 2.3 and Theorem 2.2. Suppose, on the contrary that $X$ admits a monotone map $f: X \rightarrow X$ such that $f$ has positive topological entropy. Consider the shift homeomorphism $\sigma_{f}: \lim (X, f) \rightarrow \lim (X, f)$. Since $\sigma_{f}$ has positive topological entropy, by $[26] \lim (X, f)$ is non-Suslinean. Let

$$
\mathcal{Z}^{\prime}=\left\{Z_{\alpha}^{\prime} \mid \alpha \in \Lambda\right\}
$$

be a family of uncountable mutually disjoint nondegenerate subcontinua of $\lim _{(}(X, f)$. We may assume that there is $\epsilon_{1}>0$ such that for each $\alpha \in \Lambda$, $\operatorname{diam}\left(Z_{\alpha}^{\prime}\right) \geq 5 \epsilon_{1}$. For each $\alpha \in \Lambda$, we choose a subcontinuum $Z_{\alpha}$ of $Z_{\alpha}^{\prime}$ such that $\epsilon_{1} \leq \operatorname{diam}\left(Z_{\alpha}\right) \leq 2 \epsilon_{1}$. Put

$$
\mathcal{Z}=\left\{Z_{\alpha} \mid \alpha \in \Lambda\right\}
$$

We can choose a sufficiently large natural number $n_{1} \in \mathbb{N}$ such that the projection $p_{n_{1}}$ : $\lim _{\longleftarrow}(X, f) \rightarrow X$ is an $\epsilon_{1} / 2$-map. Note that $p_{n_{1}}^{-1}(x)(x \in X)$ is a subcontinuum such that

$$
\operatorname{diam}\left(p_{n_{1}}^{-1}(x)\right)<\epsilon_{1} / 2 .
$$

Then we see that for any $\alpha \in \Lambda$ and any finitely many points $x_{i}(i=1,2, \ldots, k)$ of $X$,

$$
\text { (*) } \quad Z_{\alpha}^{\prime}-\left[Z_{\alpha} \cup \bigcup_{i=1}^{k} p_{n_{1}}^{-1}\left(x_{i}\right)\right] \neq \emptyset,
$$

because that $Z_{\alpha}^{\prime}-Z_{\alpha}$ contains a connected set whose diameter is larger than $\epsilon_{1}$ and

$$
\left\{p_{n_{1}}^{-1}\left(x_{i}\right) \mid i=1,2, \ldots, k\right\}
$$

is a finite family of mutually disjoint closed subsets whose diameters are less than $\epsilon_{1} / 2$.

For each $\alpha, \beta \in \Lambda$, we define $\alpha \sim_{f} \beta$ provided that $p_{n_{1}}\left(Z_{\alpha}\right) \cap p_{n_{1}}\left(Z_{\beta}\right) \neq \emptyset$. Also, for each $\alpha, \beta \in \Lambda, \alpha \sim \beta$ if there is a finite sequence $\alpha=\alpha_{1}, \alpha_{2}, \ldots, \alpha_{s}=\beta$ of $\Lambda$ such that $\alpha_{i} \sim_{f} \alpha_{i+1}$ for each $i=1,2, . ., s-1$. Then the relation $\sim$ is an equivalence relation on $\Lambda$ (see the proof of Lemma 2.3). We will show that for each $\alpha \in \Lambda$, the cardinality $|[\alpha]|$ of the set $[\alpha]=\{\beta \in \Lambda \mid \alpha \sim \beta\}$ is $\leq T(X)$. Suppose, on the contrary, that $|[\alpha]| \geq m=T(X)+1$. Let $\Lambda(m)$ be any subset of $[\alpha]$ with $|\Lambda(m)|=m$. As in the proof of Lemma 2.3, we can construct a subcontinuum $A$ of $\lim _{\mathrm{As}}(X, f)$ such that $A$ is a union of $Z_{\beta}(\beta \in \Lambda(m))$ and some finitely many subontinua $p_{n_{1}}^{-1}\left(x_{i}\right)$. As in the proof of Lemma 2.3, we put

$$
H=\bigcup_{\beta \in \Lambda(m)} Z_{\beta}^{\prime} \cup A
$$


By (*), we see that $Z_{\beta}^{\prime}-A \neq \emptyset(\beta \in \Lambda(m))$. Since $\left\{Z_{\beta}^{\prime} \mid \beta \in \Lambda(m)\right\}$ is a finite family of mutually disjoint subcontinua, there is a sufficiently small positive number $\epsilon_{2}<\epsilon_{1}$ such that $d\left(Z_{\beta}^{\prime}, Z_{\beta^{\prime}}^{\prime}\right) \geq \epsilon_{2}$ for $\beta \neq \beta^{\prime}\left(\beta, \beta^{\prime} \in \Lambda(m)\right)$. Then we can choose a sufficiently large natural number $n_{2} \in \mathbb{N}$ such that the projection $p_{n_{2}}: \lim _{\swarrow}(X, f) \rightarrow X$ is an $\epsilon_{2} / 2$-map such that $p_{n_{2}}\left(Z_{\beta}^{\prime}\right)-p_{n_{2}}(A) \neq$ $\emptyset(\beta \in \Lambda(m))$. Then

$$
p_{n_{2}}(H)=\bigcup_{\beta \in \Lambda(m)} p_{n_{2}}\left(Z_{\beta}^{\prime}\right) \cup p_{n_{2}}(A)
$$

is an $m$-od in $X$, because that $\left\{p_{n_{2}}\left(Z_{\beta}^{\prime}\right) \mid \beta \in \Lambda(m)\right\}$ is a family of mutually disjoint subcontinua. This means $T(X) \geq m$. This is a contradiction.

As in the proof of Lemma 2.3, we can choose an uncountable subset $\Lambda^{\prime}$ of $\Lambda$ such that the family $\left\{p_{n_{1}}\left(Z_{\alpha}\right) \mid \alpha \in \Lambda^{\prime}\right\}$ is a family of mutually disjoint nondegenerate subcontinua of $X$. This means that $X$ is non-Suslinean. This is a contradiction. Consequently, any monotone maps on $X$ have zero topological entropy.

Finally, the following problems remain open.

Problem 2.9. Let $X$ be a hereditarily decomposable continuum with $T(X)<\infty$. Is it true that any homeomorphism (more general, monotone map) on $X$ has zero topological entropy?

Problem 2.10. Let $X$ be a Suslinean continuum. Is it true that any monotone map on $X$ has zero topological entropy? In particular, is it true that any monotone map on a rational continuum has zero topological entropy?

\section{References}

[1] M. Barge and J. Martin, Chaos, periodicity, and snakelike continua, Trans. Amer. Math. Soc. 289 (1985), no. 1, 355-365.

[2] M. Barge and B. Diamond, The dynamics of continuous maps of finite graphs through inverse limits, Trans. Amer. Math. Soc. 344 (1994), no. 2, 773-790.

[3] L. S. Block and W. A. Coppel, Dynamics in one dimension, Lecture Notes in Mathematics 1513, Springer 1992.

[4] R. Bowen, Topological entropy and axiom A, 1970 Global Analysis (Proc. Sympos. Pure Math. Vol. XIV, Berkeley, Calif., 1968) pp. 23-41 Amer. Math. Soc., Providence, R.I.

[5] U. B. Darji and Hisao Kato, Chaos and indecomposability, Advances in Mathematics, 304 (2017), 793-808 (to appear).

[6] G. W. Henderson, The pseudo-arc as an inverse limit with one binding map, Duke Math. J. 31 (1964), 421-425.

[7] W. T. Ingram, Periodic points for homeomorphisms of hereditarily decomposable chainable continua, Proc. Amer. Math. Soc. 107 (1989), no. 2, 549-553.

[8] Hisao Kato, Topological entropy of monotone maps and confluent maps on regular curves, Proceedings of the 18th Summer Conference on Topology and its Applications, Topology Proc. 28 (2004), no. 2, 587-593. 
[9] Hisao Kato, Topological entropy of maps on regular curves, Topology Appl. 154 (2007), no. 6, 1027-1031.

[10] Hisao Kato, On indecomposability and composants of chaotic continua II, Topology Appl. 158 (2011), 647-652.

[11] Hisao Kato, Topological Entropy of Piecewise Embedding Maps on Regular Curves, Ergodic Theory and Dynamical Systems, 26 (2006), 1115-1125.

[12] Hisao Kato, The nonexistence of expansive homeomorphisms of a class of continua which contains all decomposable circle-like continua, Trans. Amer. Math. Soc. 349 (1997), 36453655 .

[13] Hisao Kato, Chaos of continuum-wise expansive homeomorphisms and dynamical properties of sensitive maps of graphs, Pacific J. Math. 175 (1996), 93-116.

[14] Hisao Kato, On indecomposability and composants of chaotic continua, Fund. Math. 150 (1996), 245-253.

[15] Hisao Kato, The nonexistence of expansive homeomorphisms of chainable continua, Fund. Math. 149 (1996), 119-126.

[16] Hisao Kato, Chaotic continua of (continuum-wise) expansive homeomorphisms and chaos in the sense of Li and Yorke, Fund. Math. 145 (1994), 261-279.

[17] Hisao Kato, Concerning continuum-wise fully expansive homeomorphisms of continua, Topology Appl. 53 (1993), 239-258.

[18] Hisao Kato, Continuum-wise expansive homeomorphisms, Canad. J. Math. 45 (1993), 576598.

[19] Hisao Kato and C. Mouron, Hereditarily indecomposable compacta do not admit expansive homeomorphisms, Proc. Amer. Math. Soc. 136 (2008), 3689-3696.

[20] D. Kerr and H. Li, Independence in topological and $C^{*}$-dynamics, Math. Ann. 338 (2007), no. 4, 869-926.

[21] C. Kuratowski, Topology II, Acad. Press, New York, N. Y., 1968.

[22] J. Krasinkiewicz and P. Minc, Mappings onto indecomposable continua, Bull. Acad. Polon. Sci. Math. Astronom. Phys. 25 (1977), no. 7, 675-680.

[23] J. Llibre, M. Misiurewicz, Horseshoes, entropy and periods for graph maps, Topology 32 (1993), no. 3, 649-664.

[24] P. Minc and W. R. R. Transue, Sarkovskii's theorem for hereditarily decomposable chainable continua, Trans. Amer. Math. Soc. 315 (1989), no. 1, 173-188.

[25] C. Mouron, Positive entropy homeomorphisms of chainable continua and indecomposable subcontinua, Proc. Amer. Math. Soc. 139 (2011), no. 8, 2783-2791. 
[26] C. Mouron, Mixing sets, positive entropy homeomorphisms and non-Suslinean continua. Ergod. Th. Dynamical Sys. to appear. doi:10.1017/etds.2015.10.

[27] S. Nadler, Continuum Theory. An introduction, Monographs and Textbooks in Pure and Applied Mathematics 158, Marcel Dekker, Inc., New York, 1992.

[28] G. T. Seidler, The topological entropy of homeomorphisms on one-dimensional continua, Proc. Amer. Math. Soc. 108 (1990), no. 4, 1025-1030.

[29] X. Ye, Topological entropy of the induced maps of the inverse limits with bonding maps, Topology Appl. 67 (1995), no. 2, 113-118.

\author{
Hisao Kato \\ Institute of Mathematics \\ University of Tsukuba \\ Ibaraki, 305-8571 Japan \\ e-mail: hkato@math.tsukuba.ac.jp
}

